\title{
PSYCHIATRIA DANUBINA AND CHALLENGES OF THE COVID-19 PANDEMIC: THE ANSWER IS IN THE KNOWLEDGE, EMPATHY, COHERENCE AND GLOBAL ETHICS
}

\author{
Miro Jakovljević \\ School of Medicine, University of Zagreb, Zagreb, Croatia
}

\section{SUMMARY}

The COVID-19 pandemic crisis is cruel but crucial lesson for the future of humankind. This crisis involves very complex events, complex in its origin, its spread, its effects and its consequences at multiple levels and fields with a big impact on people's mental health. On the other side, the quality of our individual, collective and public mental health is very important for successful fighting against the COVID-19 pandemic and infodemic. For a better public and global mental health we need a common background, common values and virtues on which we agree and follow them. Knowledge, ethics, empathy, coherence, solidarity, cooperation and unity are the key factors to long-term survival and flourishing of humankind. COVID-19 pandemic and infodemic can be effectively overcome only in spirit of common ethic and mutual empathy, respect, trust and public/global cooperation sowing the seeds for humanistic self, compassionate society and empathic civilization, rather than blaming, scapegoating and xenophobia. The Psychiatria Danubina focus is on expanding the world's collective knowledge and promoting mental health through the culture of empathy and global ethics.

Key words: COVID-19 pandemic - epistemic mistrust - effective and healthy communication - culture of empathy - global ethics academic cooperation in publishing

\section{INTRODUCTION}

\author{
„Consider from what noble seed you spring: \\ You were created not to live like beasts, but for \\ pursuit of virtue and knowledge"
}

Dante: Inferno 26, 118-120

The COVID-19 pandemic and infodemic, closely intertwined and associated with many challenges put everything to the test - not only our health care systems but also our academic and scientific community, ethics, moral character and the future of humankind. It is quite clear and evident that COVID-19 is not only biomedical disease which affects only somatic health producing multi-systems disorders, but it also has detrimental effects on individual and collective mental health and all aspects of well-being (Fiorillo \& Gorwood 2020). On the other side, the quality of our individual, collective and public mental health is very important for successful fighting against the COVID-19 pandemic and infodemic. Positive mental health involves the capacity to 1. realize our abilities and cope with adverse life events (resilience and antifragility), live a life with a purpose and meaning, and make a positive contribution to our communities; 2. empathize with others (understand how others think, feel and behave) and mentalize properly (predict actions, intentions and feelings of others); form positive and creative relationships with others, and feel connected and supported; 3. experience good faith, hope, peace of mind, and solidarity; 4 . practicing ethos and freedom take responsibility for oneself and for others as appropriate. The spread of the COVID-19 virus, as well as the misinformation about it, has raised unprecedented ethical challenges at the public and global levels. It seems that what humankind needs now more than ever are global ethics for health (Kueng 1988, 2004) or global health justice, collective resilience, antifragility, empathy, trust, coherence, collaboration, and science in its purest form (Jakovljevic \& Jakovljevic 2021). Public health is inherently political for many reasons, including the frequent tension between government action to protect the health of population and respect for the autonomy (right, duty and virtue) of individuals (see Rutkow 2020). Justice, empathy, resilience, solidarity, respect, transparency and cooperativeness are not only virtues of character, but also public virtues of constitutions, political arrangements and academic communities. The COVID-19 crisis has firmly confirmed human interconnectedness and interdependence in mental health and wellness on one side and disease and illness on the other at all levels. It is increasingly important for psychiatrists to have voice in the public arena about what is happening about COVID-19 fighting, misinfodemic and in promoting public mental health. Psychiatrists are uniquely qualified to recognize and call attention to pathologic thinking, emotional response and behavior in their patients, of course with primary aim to help. The same refers also to community and society in general. Prior to the COVID-19 pandemic, public and global mental health were usually viewed as a national and international policy related to the status and quality of a country's, international and global mental health care systems. Public and global mental health are the evolving broad fields of research and practice that aim to alleviate mental illnesses through the prevention, care and treatment of mental disorders, and to promote, protect and improve the mental health of people and their communities at national, international and global levels by promoting healthy lifestyles, common and 
global ethics, empathic culture, collective resilience and coherence, and compassionate society (Collins 2020). Promotion of public and global mental health is strongly related to 5CCs: 1 . commitment to a culture of dialogue, peace and respect for all life; 2. commitment to a culture of empathy, human dignity and solidarity; 3. commitment to an episteme, culture of knowledge and truthfulness; 4. commitment to a culture of justice and universal human rights; 5 . commitment to a culture of cooperativeness, partnership and friendship. All these CCs are overlapping: Knowledge and power without empathy and ethics are ruthlessness and empathy and ethics without knowledge are folly. The national and global crisis does provide an opportunity for increasing resilience, antifragility and better society and world in general. Fighting COVID-19 crisis should be our collective hero's journey to better individual, public and global mental health, compassionate society and empathic civilization.

\section{ETHOS, EMPATHY, RESILIENCE AND COHERENCE: BRIDGING INDIVIDUAL, SOCIAL AND GLOBAL MENTAL HEALTH}

\author{
"Empathy is seeing with the eyes of \\ another, listening with the ears of another, and \\ feeling with the heart of another" \\ Alfred Adler \\ "Empathy is the oxygen breathing life into \\ the relationship between individual and other" \\ Heinz Kohut (1977)
}

The COVID-19 pandemic and syndemics are not merely biological phenomena. They are also shaped by our psychological, social, political and spiritual responses to them, from beginning to end. The most various mental changes, problems, disorders, and even a new normality (pathological normality or normal pathology) associated with COVID-19 pandemic and infodemic (epistemic hypervigilance and mistrust) have been reported. There is no health without mental health; but also there is no mental health without resilience, coherence, cooperative mindset, common ethics and compassionate community. Mental health is a "neuropsychocultural construction" based on nature and nurture intertwined in irrevocable ways overcoming mindless neuroscience and brainless psychiatry (Lolas 2016). Public health involves "the science, art and practice of protecting and improving the mental health of people and their communities... Resilience, coherence, empathy and global ethic are relatively new multidimensional, but closely connected and overlapping sociopsychobiospiritual concepts, essential for the understanding of salutogenesis and pathogenesis as well as for public and global mental health (Jakovljevic 2018). The good news is that we can learn, train and improve our empathy, resilience, coherence, cooperative compassion mindset and global ethics. Effective response to COVID-19 is related to sowing the seeds for humanistic self and empathic civilization, rather than blaming, scapegoating and xenophobia.
Ethics that transcends narrow definitions of group, class and regional interests has an indispensable and important role to play in the fighting against COVID19. The effective multilateral, multidimensional and multilevel system of response to COVID-19 pandemic is not possible without a common basic human moral attitudes, epistemic trust and binding values acceptable to all in our world of differences, confusions and conflicts. COVID-19 infodemic reflects the moral empathy gap and epistemic mistrust when people do not respect the others' perspective and do not understand the moral foundations that motivate them. As empathy is the developmental core of morality, education for culture of empathy can help bridge the moral empathy gap. At present COVID-19 crisis, we need culture of empathy, epistemic trust and global ethics more than ever.

The very fundamental fact about human beings is that we are a species that has evolved to thrive on mentalization, love, kindness, compassion, and altruism associated with our interconnectedness and interdependency (see Jakovljevic \& Tomic 2016, Jakovljevic 2018). Compassion (Latin: compati - suffer with, compassio - co-suffering) and empathy (the Greek: em in or to go into; pathos - feeling), although hailed as answers to the challenges of our world, are defined in many ways. They are overlapping concepts with some differences complementing each other, and that's why we need both. Sad to say, some research has indicated that levels of empathy are falling, for example among American student fell by $48 \%$ between 1979 and 2009 (https://www.zurich.com. Empathy is the invisible force that holds humans together which is very important for survival. Empathy leads to healthy, creative, flourishing, and well-functioning families, communities, nations, societies, and civilizations. It is very important in the dialogue, peace-making, negotiation, mediation, education and cooperation. Human beings are biologically wired to need connection, attachment, recognition, validation, and belonging. Empathy is what enables us to extend our social affiliations and connect with other people in larger social, political, economic, and religious units, blocs, and cultures. It involves empathy for difference and openness to diversity. Education for love, empathy, and compassion are pillars and foundations of a better future for all. Compassionate society as the final and the noblest result of empathy is an esprit de corps of the empathic civilization of love, trust and peace.

The choice between the clash of civilizations or dialogue among them leading to the empathic global civilization of love is the most fundamental issue from the public and global mental health perspective (see Jakovljevic 2017). Empathy in human relationships is not something ready-made. It begins as an idea or principle which human being must somehow develop and live up to in their behavior and communication. Establishing a connection between empathy, mirror neurons and brain neuroplasticity indicates that empathy, mentalization, and emotion-sharing abilities as qualities can be learnt. Empathy has cognitive, emo- 
tional, behavioral and narrative dimensions so that we can speak about cognitive empathy, emotional empathy, behavioral (compassionate) and narrative empathy (see Rahman 2016). Cognitive empathy is the ability to recognize and understand how and what other people are feeling, ability to see situations from their perspective. Emotional empathy is the capability to feel what and how other people are feeling. Compassionate empathy is desire and readiness to help other people deal with their problems and emotions. Trust is usually established when people understand, empathize, wish, and try to help each other. Narrative empathy represents the one's ability to enter and tune another's frame of reference, mental model or inner/mental world. Empathy is an essential determinant of moral behavior and a fundamental corner stone in creating moral communities and global ethics. Unfortunately, empathy can be misused to induce unethical behavior and calls for riot (Council of Europe Portal 2021).

From the public mental health point community resilience is crucial to disaster preparedness, response, and recovery. It stress collective responsibility for mental health, its protection and prevention of mental distress and disorders; recognizes the key role of the state and institutions; emphasis partnerships with all those who contribute to the health and well-being of the population. Resilience may be defined as a key component of individual and collective well-being related to the ability to obtain positive outcome despite adversity at personal, public and global level (Jakovljevic 2017, 2018). It represents the capacity to adapt to stress or adversity and maintain, or even improve healthy mental, physical, social and spiritual functioning and sense of coherence. At individual level resilience is about the whole person, it includes biological, psychological, social and spiritual dimension of human existence and enables individuals and communities not only to survive and adapt to challenges and adversities but also to be better off and to grow and thrive (post-traumatic growth) in addition to overcoming a specific adversity. It includes positive transformation and personal growth, an indivisible part of mental health and health in general, well-being and quality of life as well as of recovery and treatment outcome. It is very important to note that "some resilience factors contribute to the development of other resilience factors, and, in consistency with a cascade model, together they contribute to predict personal recovery. Resilience training can result in augmented neuroplasticity and balance of neural circuits that modulate reward and motivation, emotion regulation, cognitive reappraisal and executive function, novelty seeking, harm avoidance and fear response, self-directedness, cooperativeness and adaptive social behavior, and self-transcendence. By practicing compassion, empathy and coherence we can become more resilient and antifragile and increase our overall individual and collective wellness and mental health. In facing stress or adversity an individual or a community with a strong sense of coherence will 1.wish to, be motivated to, cope (meaningfulness); believe that the challenge or problem is understood (comprehensibility); and believe that resources to cope are available (manageability) - (Antonovsky 1996). Resilient and anti-fragile communities tend to foster a culture of the compassionate mind (see Gilbert 2013) with shared values and virtues, a shared mission or goal, open and transformative leadership, use of consensus, not force, enjoyment of work, friendly atmosphere, a safe learning environment, and honest and authentic conversations.

The sense of coherence (SOC) is salutogenic concept that refers to a mental model of viewing the world which enables successful and creative coping with adversities (Jakovljevic 2018). The mental model involving the SOC is characterized by comprehensibility (cognitive component - epistemic trust: considering different perspectives and connections stimuli and processes are perceived as structured, explicable and predictable), manageability (instrumental component - appropriate resources for successful coping and dialectic balance of positive and negative elements), and meaningfulness (motivational component - challenges are worth of investing in and engaging with). Coherence implies order, structure and efficient utilization of energy and functioning and denotes alignment within and amongst systems, whether small particles, organs, individuals, families, societies, galaxies or cosmos. Generally speaking, coherence always refers to the wholeness, where the whole means more than the simple sum of its individual parts, and implies integration, synchronization, connectedness, correlations, consistency, and efficient energy utilization. From the mental health perspective, coherence is always about the whole person, it includes the biological, psychological, social, and spiritual dimension of human existence.

\section{THE ACADEMIC COMMUNICATION AND PUBLISHING: THE NEED FOR EFFECTIVE LEADERSHIP AND COMMUNICATION STRATEGIES IN GLOBAL ETHICS, HUMANITY AND EMPATHY}

\author{
"There is a nobility in compassion, a \\ beauty in empathy, a grace in forgiveness", \\ John Connolly \\ "All wars derive from lack of empathy: \\ the incapacity of one to understand and \\ accept the likeness or difference of another" \\ Marya Mannes
}

Rarely in history of the world has there been such a need for reliable academic communication and public relations as exists today. Publishing as an important form of public communication is a form of power and influence, and it carries with it heavy ethical and epistemic responsibilities (Lucas 2001). Humanistic academic communication has universal claims: it is based on a shared human nature and shared universal ethos and episteme, human dignity and universal human rights (Kueng 2004). Effective, timely and coherent communication keeping people informed, empathic and rational, 
Table 1. Elements and principles of effective communication strategies (OHA 2021 modified https://www.oha.com

Empathy: Do - Acknowledge concerns, hardship and express understanding and gratitude. Avoid: Shaming, blaming and scapegoating people and organizations

Trust and Credibility: Do - Acknowledge uncertainty and explain what is known and what is unknown. Be honest and transparent. Explain what actions are being taken and why. Employ mechanisms of accountability. Rely on experts in the field and competent messengers. Be consistent in messaging, use simple messages and correct misinformation. Emphasize personal and public relevance. Avoid over reassurance, fostering unrealistic expectations and drawing to much attention to misinformation

Autonomy and Empowerment: Do - Give people choice within a set of guidelines/principles. Express confidence in people's ability. Give people things to do. Provide specific descriptions desired behaviors. Avoid: Being paternalistic and overly authoritarian. Implying that the facts are too difficult to understand

Values, Emotions, and Stories: Do: Focus on messages of solidarity, kindness and love. Appeal to "collective" good. Link behaviors to people's identities. Focus on people adopting desirable behavior. Outline/stories that contextualize risk. Respect cultural beliefs/values. Avoid: Drawing attentions to undesirable behaviors. Appealing to fear.

Militaristic analogies/metaphors that may breed fear and xenophobic sentiment

Speed: Do: Communicate accurate information as early as possible. Acknowledge that early information may change. Avoid. Allowing misinformation and rumor to fill information void

Public Involvement and Audience Segmentation: Do: Engage public in raising awareness. Use messengers trusted by target audience. Amplify public voices. Make messages sensitive to demographics of intended target

Institutionalization: Do: Depolitize health communication. Create a pandemic communication unit. Limit the number of institutions/people delivering messages

and resilient in our COVID-19 pandemic is vital for successful dealing with COVID-19 infodemic and epistemic hypervigilance and mistrust. Unlike previous pandemics, people today want more influence and voice in deciding issues that affect their welfare, rights, duties and everyday life. We are more interconnected than ever through different media and channels and nobody is immune to misinformation. People do not accept rules by threat, fear, or political and economic stick nor rigid paternalistic control of their behavior. Effective risk communication is proactive, empathic, imaginative, creative, innovative, polite, transparent, energizing, empowering, and technology friendly (Reddy \& Gupta 2020). Empathy is a lynchpin for good academic leadership and a compassionate academic culture where leaders and members of academic community act to understand, help and support each other. According OHA 2021 there is no 'best practice' for communication during a complex health emergency, but past experience has led to several principles that contribute to a successful strategy (see table 1).

From the perspective of public relations we live in the misinfodemic world of empathic strangers divided into good guys and bad guys, people are commonly under massive media manipulation and "we-you-they" schisms as moral strangers and epistemic strangers (Lolas 2016, Jakovljevic et al. 2020, Jakovljevic \& Jakovljevic 2021). It is of crucial relevance to avoid mistakes during communication such as mixed or late messages, "father-know-best" paternalism, ignoring rumors, unrealistic expectations, power games, hubris, blaming others and scapegoating (Reddy \& Gupta 2020). In blame games participants find themselves in a vicious cycle of attack and defense and waste a lot of time and energy in useless dispute and arguing instead inventing options for mutual gain. As two persons can be using the same word, meaning different things (Taleb 2019) according to the soprosyne concept and ethics of differences it is foolish to blame or attack others due to just not understanding them. Soprosyne (ancient Greek sophrosune: excellence of character and soundness of mind) is a state, a number of qualities such as having self-control, self-knowledge, forethought and being slow to anger and blame, having restraint, having reason which create a life of harmony, of peace and of happiness. For Socrates soprosyne is strongly related to the rationality and knowledge about the nature and essence of human life (see Predanocyova 2016). Trust, empathy, respect, integrity (being honest and having strong moral principles) and coherence complementary to each other are key factors in building effective understanding, communication, and healthy and creative relationships in all human communities involving academic ones.

Hodegetics, that is a term from pedagogy, but as medical hodegetics was a discipline of medical studies still in the Middle Ages (see Jakovljevic et al. 2019), literally means signposting (old Greek: hodos - way, guidance, indicator of the way, pointing the way). Scientific publishing hodegetics is about the meaning, purpose and method of publishing scientific papers. In academic community and publishing we need epistemic hodegetics of knowledge, humanity, empathy and coherence. The systems thinking involve looking at the bigger or whole picture and understanding how all the 
parts are interconnected and interdependent. In order to produce positive change at any level, it is necessary to identify all the participants who or processes which are contributing to or are affected by some issue. Empathy and global ethic are background of systems thinking in academic community which help us to understand and resolve the problems creatively. Namely, the truth and knowledge are very important, as well as each conversation in academic community should involve empathy, understanding and coherence if we really want "to work together to unlock its (academic publishing) true potential (RG Portal 2021). We need to shift from a destructive blaming type of thinking to a much more creative and humanistic type. The art and practice of learning organizations indicate that blaming others commonly takes time and energy away from improving yourself. According to Deepak Chopra when we blame and criticize others, we commonly avoid some truth about ourselves. In academic community we should practice communication without blaming each other. When we respect and try to understand others we will be respected by them in return. Our ability to put the greater good ahead of personal desires, envies, conflicts, and blame games helps us to be problem solvers, unifiers and collaborators. Without that capacity, we'll never tackle plagiarism and predation, two important problems in scientific publishing (see Masic 2021) commonly associated with "publish or perish" concept. The National Communication Association (1999) in its Credo of Ethical Communication "condemns communication that degrades individuals and humanity through distortion, intimidation, coercion, and violence, and through the expressionof intolerance and hatred (https://courses/lumenlearning.com). Stress within academic community is up-surging and its far-reaching impacts continue to unfold worldwide (Gautam \& Sharma 2021). The origin of distress in scientific publishing community is in addition to "publish or perish" mindset related to dark tetrad personalities (see Rehman et al. 2021)

\section{PSYCHIATRIA DANUBINA MISSIONS AND ITS FAMILY JOURNALS}

\author{
It's easy to blame, it's easy to \\ politicize, it's harder to tackle a problem \\ together and find solution together \\ Tedros Adhanom Ghebreyesus
}

In the early 1980s Psychiatria Danubina (PD) was conceived primarily to bring lectures presented at the Danube Symposia of Psychiatry which have been held from 1964 on every other year in a different country of the Danube region from both side of the Iron Curtain as well as to publish lectures from the Linz Psychiatric Saturday and lectures from the Symposia of Neuropsychiatry in Pula. Contributions from other parts of the world were also welcome. Today, PD provides a new venue for promotion of personal, public and global mental health within the context of compassionate society, global ethics and empathic civilization.
The journal mission is to serve to academic community in the field of psychiatry, mental health sciences and public and global mental health in transdisciplinary and integrative way by:

- disseminating original research, concepts and theories as well as promoting creativity and cooperation in and between psychiatry, all mental health sciences and humanity disciplines;

- presenting important news from the mental health sciences and psychiatry of the Danube region countries as well as from the whole world for public and global mental health improvement, well-being and development of compassionate society and empathic civilization;

- connecting different disciplines within psychiatry to each other in new dialectical and trialectical ways to bring much more knowledge about salutogenesis and psychopathogenesis, mental health and mental disorders and its treatment and prevention;

- promoting cooperation between psychiatrists, mental health scientists and practitioners to connect their brains and knowledge through joint scientific, educational and organizational projects in the Danube region countries;

- supporting a new vistas of human capability and creativity achievable through collective intelligence, global ethics and collaborative academic development of a new academic generation from South East Europe in a regional and European context.

We prefer the authors to communicate with each other through creative interaction promoting some kind of mental health noosphere based on epistemics, art and practice of learning organizations. "Psychiatry for the Better World", "The Greatest Good for the Greatest Number" and "Serving a Purpose Larger than Yourself" are the important principles of our editorial policy. We particularly welcome papers with transdisciplinary integrative approach and with arching concepts and paradigms that unify all psychiatric disciplines and mental health sciences. PD supports publication, academic debate on the basis of art and practice of learning organization through forum, commentaries or letters to the editor. We encourage correspondence and constructive, critical, dialectic, trialectic systems thinking regarding the published articles, and that is one of the reasons why we publish supplements related to scientific conferences and congresses.

\section{The special editions of Psychiatria Danubina: "Medicina, Academica Mostariensia" and "Science, Art and Religion"}

According to Lolas (2016) we live in the world of "moral strangers" and "epistemic strangers" because people do not believe in the same things and understand them differently, even if similar words are used. Nobody is immune to misunderstanding which may fuel disagreements and unnecessary conflicts. Disagreements 
are commonly about one of three things - where we are starting from, where we want to get to, and how to get there. As special editions of Psychiatria Danubina (PD) related to Bosnia and Herzegovina were recently mentioned in an inappropriate way (see Masic 2021), it seems reasonable to explain shortly our point of view. The PD focus is on expanding the world's collective knowledge and promoting mental health through empathy, coherence and fairness in academic communication and publishing. Our "Hero's Journey (see Jakovljevic 2020, Jakovljevic \& Kurjak 2021) in Bosnia and Herzegovina involves three main interconnected goals: 1. promoting mental health sciences and helping in healing old wounds and resolution of conflicting complexes; 2 . promoting international scientific projects (Dzubur-Kulenovic et al. 2016, Jakovljevic 2019), and 3. supporting $\mathrm{PhD}$ studies.

Guiding principles of the PD across the boundaries and frontiers are to promote synergy between psychiatry, other fields of medicine and humanistic disciplines as well as to foster cooperation between all the three constitutive nations to promote public mental health through the concept of compassionate society and empathic civilization in unified Bosnia and Herzegovina. A usual statement of purpose for some project needs three parts: the goals we want to create, commitment and metrics. The general goal of our special editions is a horizon of the learning organization, culture of dialogue, systems thinking and a breadth of vision of synergy and cooperation which we want and hope to have built at each critical milestone and at the end of our Hero's Journey. The second part is a deep commitment to the goals we want to create, and transform the vision into reality. The third part is to develop metrics by which we can measure our progress and keep us moving forward together on our Hero's Journey. Following the WHO phrase "there is no health without mental health" from 2013 we have published Medicina Academica Mostariensia (MAM) as a special edition of the PD in the form of the supplement in order to support increasing the scientific level of the School of Medicine, University of Mostar. The final goal is that MAM become separated journal in the family of the PD journals. The time is ripe that after 9 years MAM in 2022 become a separated journal of the University of Mostar as a member of PD family.

Our world of ethical relativism epistemic hypervigilance, individualism, selfishness, global rivalries, free market where everything can be commodity seems to be floating over the abyss, in-between contradictions, instabilities and balance, war and peace, hatred and love, clash of civilizations and empathic civilization, dark anti-Utopia an Utopia... (Jakovljevic \& Jakovljevic 2021). In a short story entitled "A Leter from 1920" (published in 1946) Nobel Prizer Ivo Andric wrote: "Bosnia is wonderful country, fascinating, with nothing ordinary in the habitat or people. And just there are mineral riches under the earth in Bosnia, so undoubtedly are Bosnians rich in hidden moral values, which are more rarely found in their compatriots in other Yugoslav lands.
But, you see, there is one thing that the people of Bosnia, at least people of your kind, must realize and never lose sight of - Bosnia is a country of hatred and fear. Hatred which sets man against man and casts both into misery and misfortune, on drives both opponents to the grave, ; hatred like a cancer in an organism, consuming and eating up everything around it...". Unfortunately in 1990s former Yugoslavia wars Bosnia and Herzegovina really became a dark villa-yet, a country of hatred and fear. Sometimes it seems that people in Bosnia and Herzegovina live in the world of "moral strangers" and "epistemic strangers" because they do not believe in the same things and understand them differently, even if similar words are used.

Brotherhood in humanity is a life philosophy that all human beings belong to a single family/community based on shared logos, thelos, pathos, ethos and mythos. Bosnia and Herzegovina is a right place for spiritually integrated sciences, arts and religions in creative dialogues as allies to significantly contribute to promoting compassionate society and empathic civilization. Having published the special edition "Science, Art and Religion" (SAR) as a supplement, the PD has supported the establishing International Academy of Science, and Art in Bosnia \& Herzegovina (IASAB\&H) in May 2021 (Jakovljevic \& Kurjak 2021). The SAR, as an official journal of the IASAB\&H) will be published by the Jaypee Brothers Medical Publishers as a separated journal in the very near future.

\section{CONCLUSIONS}

The present COVID-19 pandemic and epidemic mistrust has raised a number of questions about the importance and roles of psychiatry and mental health sciences in medicine and society. It seems that fundamental changes are rolling on the horizon with regards to future psychiatry in terms how it will be implemented and how we are going to establish mental health sciences in practice. Empathic civilization is endowed with moral guidelines for human behavior which involve a common, universal standard of values, ideals and goals. Unifying values and norms are becoming more and more important in the age of COVID-19 pandemic and infodemic. Psychiatria Danubina promotes transdisciplinary integrative psychiatry which has been devoted to the three missions: 1. to study, diagnose, treat and prevent mental disorders; 2 . to help other branches of medicine to be more successful and to promote person-centered medicine through practice of the consultation-liaison psychiatry; and 3. to promote public and global mental health and the concepts of compassionate society and empathic civilization.

\section{Acknowledgements: None.}

Conflict of interest: None to declare. 


\section{References}

1. Andric I: Letter from 1920. http://diogen.weebly.com

2. Antonovsky A: The salutogenic model as a theory to guide health promotion. Health Promotion International 1996; 11:11-18

3. Collins PY: What is global mental health? World Psychiatry 2020; 19:265-266 doi: 10.1002/wps.20728

4. Council of Europe Portal: Ethics and Empathy. https://coe.int

5. Dzubur-Kulenovic A, Agani F, Avdibegovic E, Jakovljevic M, Babic D, Kucukalic A, Deckert J: Molecular mechanisms of posttraumatic stress disorder (PTSD) as a basis for individualized and personalized therapy: Rationale, design and methods of the south eastern europe (SEE)PTSD study. Psychiatr Danub 2016: 28:154-163. Retrieved from www.scopus.com

6. Fiorillo $A \&$ Gorwood P: The consequences of the COVID19 pandemic on mental health and implications for clinical practice. European Psychiatry 2020; 63:1-4. doi:10.1192/j.europsy.2020.35

7. Gautam $R$ \& Sharma M: Consequences of stress on academic fraternity: A meta-analytical review. Psychiatr Danub 2021; 33(Suppl 10):S3-17 - (special edition: Medicina Academica Mostariensia 2021; 9:106-108)

8. Gilbert P: The Compassionate Mind. Constable \& Robinson, London 2013

9. Jakovljevic M\& Tomic Z: Global and public mental health promotion for empathic civilization: The role of political psychocultures. Psychiatria Danubina 2016; 28:323-333

10. Jakovljevic M: Resilience, psychiatry, and religion from public and global mental health - From divide to dialogue and cooperation in the search for humanistic self, compassionate society and empathic civilization. Psychiatr Danub 2017; 29:238-244 https://doi.org/10.24869/psyd.2017.238

11. Jakovljevic M: Empathy, sense of coherence and resilience: Bridging personal, public and global mental health and conceptual synthesis. Psychiatr Danub 2018; 30:380-384

12. Jakovljevic M, Masic I, Ostojic Lj, Bevanda M \& Begic E: Medical hodegetics - Almost forgotten art and science of upbringing medical doctors. Psychiatr Danub 2019; 31(Suppl 1):S2-S8 - special edition Medicina Academica Mostariensia 2018; 6:2-8

13. Jakovljevic $M \&$ Borovecki F: Epigenetic, resilience, comorbidity and treatment outcome. Psychiatr Danub 2018;30:242-253 https://doi.org/10.24869/psyd.2018.242

14. Jakovljevic M: In search for biomarkers, endophenotypes or biosignature of PTSD: What have we learned from the South East European Study. Psychiatr Danub 2019; 3:282-9

15. Jakovljevic M: COVID-19 crisis as a collective hero's journey to better public and global mental health. Psychiatr Danub 2020; 32:3-5 https://doi.org/10.24869/psyd-2020.3

16. Jakovljevic M, Bjedov S, Jaksic N \& Jakovljevic I: COVID19 pandemia and public and global mental health from the perspective of global health security. Psychiatr Danub 2020; 32:6-14. https://doi.org/10.24869/psyd.2020.6
17. Jakovljević M, Bjedov S, Mustac F \& Jakovljevic I; COVID19 infodemic and public trust from the perspective of public and global mental health. Psychiatr Danub 2020; 32:449457. https://doi.org/10.24869/psyd.2020.449

18. Jakovljevic $M$ \& Kurjak A: Where science meets humanity: The three good reasons to add religion in academy. Psychiatr Danub 2021 (suppl 3); 33:S235-S237 - special edition Science, Art and Religion 2021; 1:2-4

19. Jakovljevic $M \&$ Jakovljevic I: Sciences, arts, and religions: The triad in action for empathic civilization in Bosnia and Herzegovina. Psychiatr Danub 2021; (Suppl 3):33:S235-52 special edition Science, Art and Religion 2021; 1:5-22

20. Khaimook Laopipattana: The Imperative of a Global Ethics in a Global Society. Mahachula Academic Journals 2020; 7:301-314 https://so04.tci-thaijo.org

21. Kueng H: A Global Ethic for Global Politics and Economics. Oxford University Press, 1988

22. Kueng H: Global Responsibility: In Search of a New World Ethic. Wipf and Stock Publishers, 2004

23. Lolas F: Global mental health: Challenges for a global ethics. Acta Bioethica 2016; 22:9-14.

24. 24.Lucas SE: The Art of Public Speaking (7th edition). McGraw Hill, 2001 see https://courses/lumenlearning.com

25. Masic I: Predatory Journals and Publishers - Dilemmas: How to Assess it and How to Avoid it? http://creativecommons-org/licenses/by-nc/4.0/

26. Ontario Hospital association (OHA): Effective Communication Strategies for COVID-19 - Research brief. https://www.oha.com

27. Predanocyova L: Socrates view on sophrosyne. Syncronia 2016, No 69. https://www.redalyc.org/articulo.oa? id= 513852378009

28. RahmanWAWA: Empathy and trust: Into a better workplace environment. Journal of Bussiness and Economics 2016; 7:2025-2034

29. Reddy B V \& Gupta A: Importance of effective communication during COVID-19 infodemic. Journal of Family Medicine and Primary Care 2020; 9:3793-6. http://www.jfmpc.com

30. Rehman S, Laila U, Rothmann S \& Naz S: Dark tetrad personality during COVID-19: An overview of psycho-somatic therapies for managing mental health response. Psychiatr Danub 2021; 33(Suppl 10):S106-108 (special edition: Medicina Academica Mostariensia 2021; 9:106-108)

31. RG Portal: A note on recent content takedowns. Available at: https://www.researchgate.net/blog/post/a-note-on-recentcontent-take-downs?fbclid $=I w A R 3 \mathrm{cWbMmKtyhYN761M6l}$ DlOV pwdrStor4Uc9g-bHe_J8PeGhbYp_MCWPB8uk

32. Rutkow L: Origins of the COVID-19 pandemic and the path forward. In Brands $H$ \& Gavin FJ (eds): COVID-19 and World Order - The Future of Conflict, Competition, and Cooperation, 93-113. John Hopkins University Press, 2020. Project MUSE. doi:10.1353/book.77593

33. Taleb NN: Skin in the Game: Hidden Asymmetries in Daily Life. Penguin Random House, UK, 2019

Correspondence:

Professor Miro Jakovljevic, $M D, P h D$

School of Medicine, University of Zagreb

Salata 3, 10000 Zagreb, Croatia

E-mail: jakovljevic.miro@yahoo.com 